\title{
Prognostic Factors Determining Survival of Patients Operated for Non-Small Cell Lung Cancer with Consideration Given to Morphological Parameters of Blood
}

This article was published in the following Dove Press journal:

Cancer Management and Research

\author{
Mariusz Łochowski ${ }^{1}$ \\ Barbara Łochowska ${ }^{2}$ \\ Justyna Chałubińska- \\ Fendler $\left(\mathbb{D}^{3}\right.$ \\ Izabela Zawadzka ${ }^{4}$ \\ Marek Rębowski' \\ Józef Kozak'
}

'Clinic of Thoracic Surgery and Respiratory Rehabilitation, Medical University of Lodz, Regional MultiSpecialist Center for Oncology and Traumatology of the Nicolaus Copernicus Memorial Hospital in Lodz, Lodz, Poland;

${ }^{2}$ Department of Radiotherapy and General Oncology, Regional MultiSpecialist Center for Oncology and Traumatology of the Nicolaus Copernicus Memorial Hospital in Lodz, Lodz, Poland; ${ }^{3}$ Department of Radiotherapy, Military Institute of Medicine, Warsaw, Poland; 4"Synevo" Medical Laboratory, Regional Multi-Specialist Center for Oncology and Traumatology of the Nicolaus Copernicus Memorial Hospital in Lodz, Lodz, Poland

Correspondence: Mariusz Łochowski Department of Thoracic Surgery, Medical University of Lodz, UI. Pabianicka 62, Lodz 93-5 I3, Poland

Tel +484268952 31

Email marilo@op.pl
Introduction: Prognostic biomarkers are the area of high interest in non-small cell lung cancer (NSCLC). Inflammatory blood markers can be routinely determined from complete blood counts which are inexpensive and reliable. The aim of the study was to determine prognostic parameters which, in early diagnostics, best determine survival of patients, operated on due to NSCLC.

Materials: The study was conducted on 532 (174 females and 358 males) patients, operated on due to NSCLC, in stages IA - III, aged 36-84 years (the mean age: 63.6 years). The following parameters were subjected to a statistical analysis, conducted in order to determine prognostic values of the number of leukocytes, neutrophils, monocytes, platelets, haemoglobin, RDW-CV and MCV, calculated values of PLR, NLR, and LMR ratios, age, sex, smoking, histopathological diagnosis, $\mathrm{T}$ stage, $\mathrm{N}$ stage, the Charlson Comorbidity Index (CCI), type of surgery, and potential complications.

Results: The univariate analysis revealed an impact of NLR, PLR, and LMR values, RDWCW and CCI ranges, and also the number of monocytes on patients' overall survival (OS). The multivariate analysis identified six independent negative prognostic factors: male sex (0.001), CCI $>4(p=0.000007)$, RDW-CV $>14.5 \%$ and PLR $>144(p=0.000001, p=0.001$, respectively), the number of metastatic N2 lymphatic nodes $(\mathrm{p}=0.0003)$, and existence of post-operative complications $(\mathrm{p}=0.008)$.

Conclusion: Patients' sex, RDW and PLR values, Charlson index, the number of involved $\mathrm{N} 2$ nodes by cancer and postoperative complications are independent and significant prognostic factors in patients operated on due to NSCLC.

Keywords: carcinoma, non-small cell, comorbidity index, red cell distribution width, platelets, lymphocytes

\section{Introduction}

Recently a dramatic increase in the incidence of malignancies is observed worldwide. $^{1}$

Before smoking nicotine became a common habit, lung cancer had occurred extremely rarely. In 1911, doctor Issac Adler reported in literature only 374 cases of this disease. ${ }^{2}$ In 2012, over 1.6 million people died of lung cancer worldwide. It is estimated that in by year 2035 , as many as 3 million people $(2.1$ million men and 0.9 million women) will have died due to this kind of cancer. ${ }^{1}$ Considering poor 
prognosis of this cancer, many oncological studies focus on a search for markers for diagnostic and prognostic purpose.

A prognostic factor by definition is a measurement associated with outcome regardless of the initiated treatment method or along with standard therapy performed in particular case. ${ }^{3}$ Common prognostic factors of a neoplastic disease include its advanced stage, metastases in lymphatic nodes and distant organs. Identifying independent prognostic factors could provide an extra value on prognosis and therapy among early or advanced stages of a disease. Researchers seek various laboratory parameters, more specific to cancer or cancer microenvironment, e.g., biochemical proteins, such as CEA or CYFRA 21.1/cytokeratin-19, but also those which are indicators of disturbances of systemic haemostasis, being a result of a trauma caused by the cancer disease., ${ }^{4,5}$

The authors of this article focused on an analysis of parameters available in a panel of laboratory tests, made in patients prior to a surgery and their association with the patients' clinical data.

The aim of the study was to determine prognostic factors based on common blood tests in patients with non-small cell lung cancer (NSCLC) who are being qualified for surgery.

\section{Materials}

\section{Selection of Patients}

In 2007-2014, 1013 patients were treated surgically due to lung malignancies. Before the operation, all of the patients had undergone chest computed tomography (CT) and bronchoscopy as well as an ultrasound of the abdominal cavity and positron-emission tomography with CT (PETCT) examination. Lymph nodes were collected via mediastinoscopy and from 2008, and endobronchial ultrasound (EBUS) examinations were also performed. Those patients, who did not demonstrate metastases to lymph nodes or in whom the neoplastic process invaded only one group of nodes and the nodes were not bulky (PET-CT), were qualified for surgery. Upon admission to hospital, all the patients underwent basic laboratory tests required for the surgery, with the use of a flow cytometer (Sysmex XN 2000, Sysmex Europe GmbH, Norderstedt, Germany).

\section{Patients' Clinical Characteristics}

Five hundred and thirty-two patients, operated in 2007-2014 due to NSCLC, were included in analysis. We excluded from the study: patients with carcinoid and patients, who had undergone wedge resections or segmentectomies. The Bioethics Committee at The Medical University gave its consent for this study to be conducted with No: RNN/83/ $19 /$ KE. All patients provided informed consent, and that this study was conducted in accordance with the Declaration of Helsinki. There were 358 males and 174 females, aged 36-84 years (the mean age: 63.6 years), of whom 295 (55\%) admitted to a nicotine addiction. According to the Charlson Comorbidity Index (CCI): 100 patients with CCI score 2-3, 375 patients with score 4-6 and 57 patients with score 7 or higher. The tumour was localized in right lung in 311 patients and in left lung in 221 patients. Histopathological reports confirmed squamous cell carcinoma in 269 cases, adenocarcinoma in 204 cases, large cell carcinoma in 43 cases and mixed carcinoma (adenosquamous carcinoma) in 16 cases - shown in detail in Table 1 .

\section{Surgical Treatment}

All the patients underwent anatomic lung resections (lobectomy, bilobectomy and pneumonectomy), performed with a standard technique, through anterolateral thoracotomy under general anaesthesia with the use of a double-lumen

Table I Patient Characteristics

\begin{tabular}{|l|l|l|}
\hline Parameters & $\mathbf{n}$ & $\%$ \\
\hline Sex & & \\
F & 174 & 33 \\
M & 358 & 67 \\
\hline Nicotinism & & \\
Yes & 295 & 55 \\
No & 237 & 45 \\
\hline Localization - lung & & \\
Left & 220 & 41 \\
Right & 312 & 59 \\
\hline Localization - lobe & & \\
Left upper & 119 & 22 \\
left lower & 101 & 19 \\
right upper & 178 & 34 \\
middle & 16 & 3 \\
right lower & 118 & 22 \\
\hline Charlson Comorbidity Index (ICC) & & \\
2 & 20 & 4 \\
3 & 80 & 15 \\
4 & 141 & 27 \\
5 & 126 & 24 \\
6 & 108 & 20 \\
7 & 43 & 8 \\
8 & 12 & 2 \\
9 & 2 & 0.004 \\
\hline
\end{tabular}


tube. In patients with involvement of the right lung, a lymphadenectomy procedure was performed for lymph nodes of the following groups according to Japan Lung Cancer Society classification. ${ }^{6}$ 2R, 3A, 4R, 7, 8, 9, 10, 11. Lymph nodes of groups: $3 \mathrm{~A}, 4 \mathrm{~L}, 5,6,7,8,9,10,11$ were removed if the left lung was involved in the neoplastic process. At least 6 node groups of N1 and N2 categories had to be dissected. The stage of tumour stage was identified on the base of the TNM Classification of Malignant Tumours (UICC 2017-8thedition), whereas the collected lymph nodes were classified in compliance with the Naruke map. ${ }^{7}$

\section{Statistical Methods}

The following parameters were subject to a statistical analysis, conducted in order to determine prognostic values of age, sex, smoking, the number of leukocytes, neutrophils, monocytes, platelets, haemoglobin, red blood cell distribution width - coefficient of variation (RDW-CV) and mean corpuscular volume of red cell (MCV) concentrations, calculated values of the platelet to lymphocyte ratio (PLR), the neutrophil to lymphocyte ratio (NLR), and the lymphocyte to monocyte ratio (LMR), histopathological diagnosis, T stage, $\mathrm{N}$ stage (including the number of metastatic lymphatic nodes from N1 and N2 categories), the CCI Index, type of surgery, complications. The NLR value was identified as a ratio of the absolute number of neutrophil to the absolute number of lymphocytes; the PLR value - as a ratio of the absolute number of platelets to the absolute number of lymphocytes, and the LMR value - as a ratio of the absolute number of lymphocytes to the absolute number of monocytes.

The authors applied the following statistical tests:

- the Shapiro-Wilk - to verify continuous variables,

- the U Mann-Whitney - to compare two groups,

- the Kruskal-Walis test with post hoc comparisons, with the application of the Dunn-Bonferroni test - to compare a larger number of groups,

- the Chi-square test for nominal variables - for nominal data,

- the Kaplan-Meyer curves test - to assess survival curves,

- Spearman Rank Correlation - for correlation assessment,

- the Log-rank test - for a univariate analysis of nominal parameters,

- the Cox proportional hazard model - for a univariate analysis of continuous parameters and a multivariate analysis of survival data

\section{Results}

\section{Surgical Treatment and Post-Operative Advancement Stage}

The most common performed procedure was lobectomy 400 cases $(75 \%)$. Pneumonectomy was performed in 94 patients (18\%), whereas - bilobectomy - in 37 patients (7\%). R0 resection was obtained in all patients. Three patients died postoperatively (one due to an infarct, one due to perforation of a gastric ulcer and one due to a stroke). Another 5 patients died within 30 days following the surgery. Complications were observed in 112 patients (21\%). In most cases, they were prolonged air leak (59 patients) and atelectasis which required interventional bronchoscopy (20 patients). Nineteen patients required postoperative transfusion of packed red blood cells and 10 patients were treated due to paroxysmal atrial fibrillation. On the base of postoperative histopathological examination, 220 patients (41\%) were diagnosed with stage I, 180 patients (34\%) - with stage II, and $132(25 \%)$ - with stage III (Table 2).

\section{Overall Survival (OS) of Patients}

The median OS of the patients was 50 months. Seventysix percent of the patients survived 1 year, $57 \%$ - 2 years, $46 \%-3$ years and $35 \%$ of the patients survived 5 years. The statistical analysis of OS revealed a significantly longer survival in females (4.87 years vs 3.91 years; $\mathrm{p}=0.00117)$ and nicotine addicts (4.63 years vs 3.88 years; $\mathrm{p}=0.006$ ). Patients with $\mathrm{T} 1$ and $\mathrm{T} 2$ local stage lived significantly longer than those staged $\mathrm{T} 3$ and $\mathrm{T} 4$ $(\mathrm{p}=0.0009)$; also, the survival was longer in those with $\mathrm{N} 0$ category than in those with $\mathrm{N} 1$ and N2 stage (respectively: 4.80 years vs 3.66 years vs 2.76 years; $p=0.0000$ ). Patients who underwent lobectomy or bilobectomy lived significantly longer than patients after pneumonectomy (4.42 years and 4.51 vs 3.23 years; $p=0.0046$ ). Tumour localization in particular lung or lobe did not contribute to statistical significance and did not significantly affect survival ( $\mathrm{p}=0.048$ and $\mathrm{p}=0.01$, respectively).

There was no significant relationship between the type of histopathological findings and the survival period $(p=0.7)$. The patients who demonstrated postoperative complications lived significantly shorter (2.11 years and 2.89 years, respectively; $p=0.008$ ). For the CCI Index, the authors calculated the Receiver Operating Characteristic (ROC), which allowed to determine the maximum values of sensitivity and specificity of the studied parameter. The 
Table 2 Pathology, Extent of Pulmonary Resection and Postoperative Complications

\begin{tabular}{|l|l|l|}
\hline Parameter & $\mathbf{n}$ & $\%$ \\
\hline Diagnosis & & \\
Squamous cell carcinoma & 269 & 50 \\
Adenocarcinoma & 204 & 38 \\
Large cell carcinoma & 42 & 8 \\
Mixed type carcinoma & 16 & 4 \\
\hline Stage of the disease & & \\
T IAI & 3 & 1 \\
T IA2 & 40 & 8 \\
T IA3 & 39 & 8 \\
T IB & 138 & 25 \\
T IIA & 8 & 2 \\
T IIB & 172 & 32 \\
T IIIA & 132 & 24 \\
\hline Surgical treatment & & \\
Lobectomy & 400 & 74 \\
Bilobetomy & 37 & 7 \\
Pneumonectomy & 94 & \\
\hline Complications & & \\
All complications & 112 & \\
Prolonged air leak & 59 & \\
Atelectasis & 20 & \\
Postoperative transfusion & 19 & \\
Atrial fibrillation & 10 & \\
Bronchia fistula & 1 & \\
Pleura empyema & \\
Pneumonia & \\
\hline
\end{tabular}

area under curve (AUC) was 0.598 (95\% CI: 543-653 $\mathrm{p}=0.0005)$ and the optimal CCI limit cut-off value was 4 (Figure 1A). The lifespan of patients with the CCI value 4 or less was significantly shorter (5.02 years vs 3.55 years; $p=0.0000$ ). ROC was calculated for the number of involved nodes from N1 and N2 stage. AUC for these parameters was, respectively, 0.533 (95\% CI: 0.494-$0.612 ; \mathrm{p}=0.0671)$ and 0.578 (95\% CI: $0.518-0.638$; $\mathrm{p}=0.0111)$. The optimal value for the number of nodes of the N1 stage was 1, and for the number of nodes of the N2 category was 2 (Figure 1B).

\section{Selected Morphological Parameters of Blood as Prognostic Factors}

ROC curves were used in order to optimally determine cutoff values for the number of lymphocytes, neutrophils, monocytes, platelets, haemoglobin, RDW-SD, MCV as well as PLR, LMR, and NLR. For lymphocytes, neutrophils, monocytes, and platelets the respective AUC values were: 0.488 (95\% CI: $0.429-0.547, \mathrm{p}=0.689)$; 0.570 (95\% CI: $0.513-0.627, \mathrm{p}=0.0659)$; 0.574 (95\% CI: $0.515-0.633, \mathrm{p}=0.0143) ; 0.568 \quad(95 \%$ CI: $0.509-0.627$, $\mathrm{p}=0.0232$ ). Optimal limit values for lymphocytes were $2.13 \times 10^{3}$, for neutrophils $6.95 \times 10^{3}$, for monocytes $0.87 \times 10^{3}$, and for platelets $259 \times 10^{3}$. AUC values for haemoglobin, RDW-CV and MCV were, respectively, 0.557 (95\% CI: 0.507-0.607, $\mathrm{p}=0.0264) ; 0.641$ (95\% CI: $0.592-0.693, \mathrm{p}=0.0001$ ); and 0.530 (95\% CI: $0.480-0.590$, $\mathrm{p}=0.0258)$. Optimal limit values for haemoglobin were 13.2, for RDW-CV 14.5\% and for MCV 89.5 (Figure 1C). For NLR, LMR and PLR, the authors calculated AUC values, which appeared to be, respectively, 0.587 (95\% CI: $0.531-0.642, \mathrm{p}=0.0024)$; 0.587 (95\% CI: $0.531-0.642$, $\mathrm{p}=0.0024)$; and 0.567 (95\% CI: 0.509-0.624, $\mathrm{p}=0.0238$ ). Optimal limit values for NLR were 2.235, for LMR 2.86 and for PLR 144.77 (Figure 1D).

We were aware that collinearity may be an issue with building accurate multivariate survival models. Therefore, we screened the variables first with univariate regression and then attempted backward stepwise multivariate model building procedure to determine which of the variables remained and which could be excluded as redundant. The final multivariate logistic regression model retained both RDW-CV and PLR - exclusion of either worsened model performance which urged us to retain them both. An identical outcome (data not shown) was obtained when we used forward stepwise variable selection - again both RDW-CV and PLR remained in the final iteration of the model convincing us that despite the variables being correlated both may have an additional, partially independent association with survival.

A univariate analysis revealed significant relationships between the values of RDW-CV, NLR, PLR, and LMR ratios, the number of monocytes and OS of the patients (Table 3).

A mulitvariate analysis for survival (including backward stepwise mulitvariate model building procedure) above 5 years revealed that in patients operated due to NSCLC, negative prognostic factors include: male sex (0.001), CCI $>4(\mathrm{p}=0.000007)$, RDW-CV $>$ $14.5 \%$ and PLR $>144.77(\mathrm{p}=0.000001$ and $\mathrm{p}=0.001$, respectively), the number of involved N2 nodes $>2$ $(p=0.0003)$ and occurrence of postoperative complications $(\mathrm{p}=0.008)$.

(Table 4). Figure 2 presents Kaplan-Meier curves of survival in the multivariate model. 

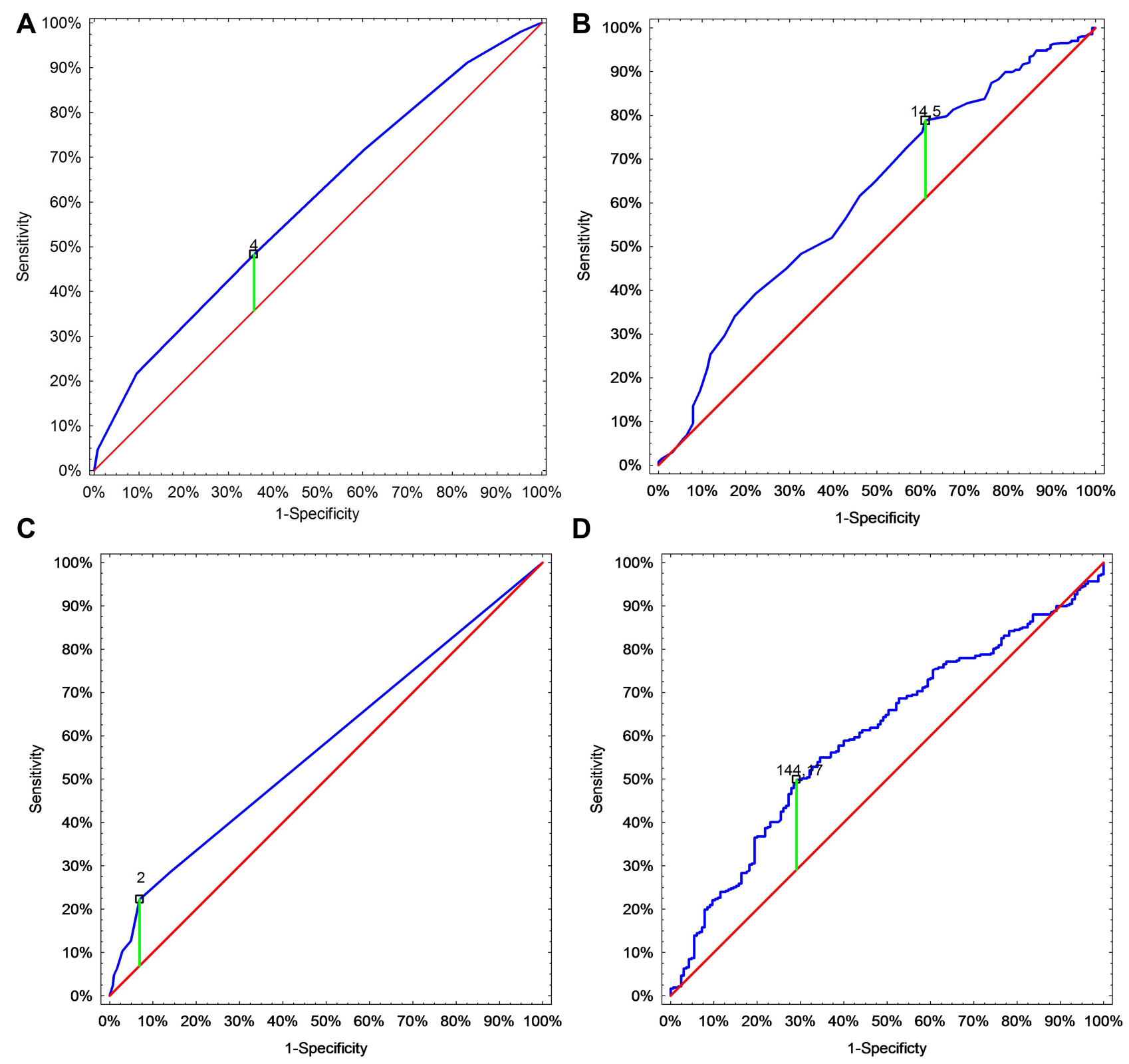

Figure I Youden index with receiver operating characteristic (ROC) curves. (A) Charlson Comorbidity Index (CCI). (B) Red cell distribution width-coefficient of variation. (C) The number of involved N2 nodules. (D) Platelet to lymphocyte ratio.

\section{Discussion}

Prognostic factors can be divided into: routinely evaluated biochemical markers, factors which are dependent on the patient, neoplastic disease, and those related to the applied treatment method. Biochemical factors evaluated in laboratory tests are haemoglobin, lactate dehydrogenase (LDH), or neutrophils. Patient-dependent factors include smoking, body weight, preoperative performance, comorbidities, age, and sex. Cancer-dependent factors include: location and advancement stage of the neoplasia as well as its histopathological type, whereas treatment parameters include choice and quality of treatment. ${ }^{3,4}$
A meta-analysis published in 2002 showed that 169 prognostic factors were identified in 176 studies. The analyses included on average 120 patients (range 31-1281 patients), multivariate analyses revealed on average 2 significant factors (range $0-6$ factors). ${ }^{8}$

Our study included 532 patients. The conducted analysis allowed to identify 6 highly significant factors, determining the patients' survival (sex, the RDW rate, the PLR ratio, the $\mathrm{CCI}$, the number of involved $\mathrm{N} 2$ nodes and occurrence of postoperative complications).

Male sex is an unfavourable, independent prognostic factor in patients with NSCLC. Our study showed that 
Table 3 Results of Studied Parameters and Univariate Analysis in Patients Operated on Due to NSCLC

\begin{tabular}{|l|l|l|l|l|l|}
\hline Parameter & Median & Range & HR & $\mathbf{9 5 \%}$ Cl & $\mathbf{P}$ \\
\hline Neutrophils $\left[\times 10^{3}\right]$ & 5.8 & $1.4-31.0$ & 1.03 & $1.01-1.04$ & 0.02 \\
Lymphocytes $\left[\times 10^{3}\right]$ & 1.9 & $0.5-9.3$ & 0.84 & $0.72-0.97$ & 0.02 \\
Monocytes $\left[\times 10^{3}\right]$ & 0.7 & $0.3-2.4$ & 1.76 & $1.25-2.48$ & 0.00 \\
Platelets $\left[\times 10^{3}\right]$ & 262.5 & $30.0-674.0$ & 1.00 & $1.00-1.00$ & 0.16 \\
RDW-CV $[\%]$ & 13.8 & $12.2-29.5$ & 1.17 & $1.11-1.22$ & 0.00 \\
Hemoglobin[g/dl] & 13.6 & $9.5-16.9$ & 1.00 & $0.96-1.04$ & 0.56 \\
MCV $[\mathrm{fL}]$ & 46.6 & $36.6-88.6$ & 1.00 & $0.98-1.02$ & 0.92 \\
NLR & 2.7 & $0.5-12.3$ & 1.05 & $1.02-1.09$ & 0.00 \\
PLR & 148.0 & $17.6-570$ & 1.00 & $1.00-1.00$ & 0.00 \\
LMR & 2.9 & $0.7-26.1$ & 0.83 & $0.76-0.89$ & 0.00 \\
\hline
\end{tabular}

Table 4 Multivariate Analysis in Patients Operated on Due to NSCLC

\begin{tabular}{|c|c|c|c|}
\hline Parameter & HR & $95 \% \mathrm{Cl}$ & $\mathbf{p}$ \\
\hline Neutrophils $\left[\times 10^{3}\right]$ & 1.145 & $0.986-|.33|$ & 0.075 \\
\hline Lymphocytes $\left[\times 10^{3}\right]$ & 1.137 & $0.643-2.012$ & 0.656 \\
\hline Monocytes $\left[\times 10^{3}\right]$ & 1.735 & $0.404-7.443$ & 0.458 \\
\hline Platelets $\left[\times 10^{3}\right]$ & 0.997 & $0.992-1.002$ & 0.366 \\
\hline RDW-CV>|4,5 [\%] & 0.908 & $0.865-0.952$ & 0.000001 \\
\hline Hemoglobin [g/dl] & 0.962 & $0.90 \mathrm{I}-\mathrm{I} .027$ & 0.248 \\
\hline MCV & 1.005 & $0.987-1.024$ & 0.925 \\
\hline$N L R>2.23$ & 0.947 & $0.713-1.395$ & 0.988 \\
\hline PLR $>144.77$ & 0.994 & $0.991-0.997$ & 0.001 \\
\hline LMR >2.86 & 1.027 & $0.739-1.427$ & 0.870 \\
\hline $\mathrm{CCl}>4$ & 0.371 & $0.247-0.559$ & 0.000007 \\
\hline Number of involved N2 nodes & 0.217 & $0.096-0.833$ & 0.0003 \\
\hline Complications & 2.205 & $1.292-3.762$ & 0.008 \\
\hline Sex (male) & 0.486 & $0.320-0.738$ & 0.001 \\
\hline Smoking & 1.257 & $0.850-1.858$ & 0.251 \\
\hline
\end{tabular}

Note: Statistically significant values are printed as bold.

men live significantly shorter after lung cancer surgery ( $\mathrm{p}$ $=0.001)$ both in univariate and multivariate analysis. Visnivesky et al observed that OS in women is longer irrespective of the administered treatment. The authors imply that lung cancer in females might have a different course. ${ }^{9}$ Similar conclusions were drawn by Ulas et al. The study conducted by them showed that non-smoking females with adenocarcinoma, and without comorbidities demonstrate a longer survival period. ${ }^{10}$

Rapid progression of a neoplastic process is perceived by an organism as a trauma and triggers an inflammatory reaction, which is accompanied by a release of different mediators of an inflammatory process. Laboratory tests reveal leukocytosis, neutrophilia, lymphocytopenia, and thrombocythemia. ${ }^{11,12}$ The neutrophil to lymphocyte ratio
(NLR) and the platelet to lymphocyte ratio (PLR) are considered reliable markers of inflammatory processes. ${ }^{13}$

A meta-analysis published by Qiang et al carried out on 1554 patients from 7 centres indicated a highly negative prognostic value of PLR in patients with NSCLC. ${ }^{12}$ The multivariate analysis which we conducted revealed that PLR values above 144, observed in our group of patients, constitute a significantly negative prognostic factor. A similar observation was made by Lee et al. They noted that PLR values above 180, advancement stages II and III according to the TNM, male sex, postoperative radiotherapy are negative and independent prognostic factors. ${ }^{14} \mathrm{Xu}$ et al - authors of another study, confirmed that with regards to T stage, PLR $>135$ is an independent prognostic factor. ${ }^{15}$ A study conducted by Toda et al revealed that the PLR ratio $>162$ in NSCLC patients treated with adjuvant postoperative chemotherapy is considered a negative prognostic factor. ${ }^{16}$

Published studies also draw attention to an important role of the NLR ratio in the event of an ongoing inflammatory process during the course of NSCLC.11,15 A meta-analysis published by $\mathrm{Yu}$ et al and performed on 7219 patients revealed that preoperative evaluation of NLR allows to identify patients with NSCLC with poor prognosis in. ${ }^{17}$ The univariate analysis in our study showed a significant impact of elevated NLR values on OS of surgically treated NSCLC patients. However, the multivariate analysis did not confirm the role of the above ratio.

Elevated values of RDW-CV also indicate an inflammatory process in the course of a neoplastic disease. $\mathrm{RDW}-\mathrm{CV}$ is a quantitative parameter and shows changes in the size of erythrocytes in the bloodstream. The parameter is applied in diagnosing various types of anaemia. ${ }^{18,19}$ The analysis which we conducted showed that $\mathrm{RDW}-\mathrm{CV}>14.5 \%(\mathrm{p}=0.000000)$ is an independent prognostic factor in patients operated due to NSCLC. Our results are not different from those obtained by other authors. Koma et al revealed that elevated RDW-CW values $(>15 \%)$ are associated with worse prognosis of patients with NSCLC $(p=0.002) .{ }^{20}$ Warwick et al based their study on 917 patients operated due to NSCLC. The authors noted that preoperative RDW-CW values above $15.3 \%$ are a significant risk factor of post-operative mortality $(\mathrm{p}=0.001)$ and survival period $(\mathrm{p}=0.0001){ }^{21}$ A similar size group of patients (992) was subject to an analysis, made by Ichinose et al They noted that preoperative RDW-CV $>15 \%$ in patients above the age of 75 years 

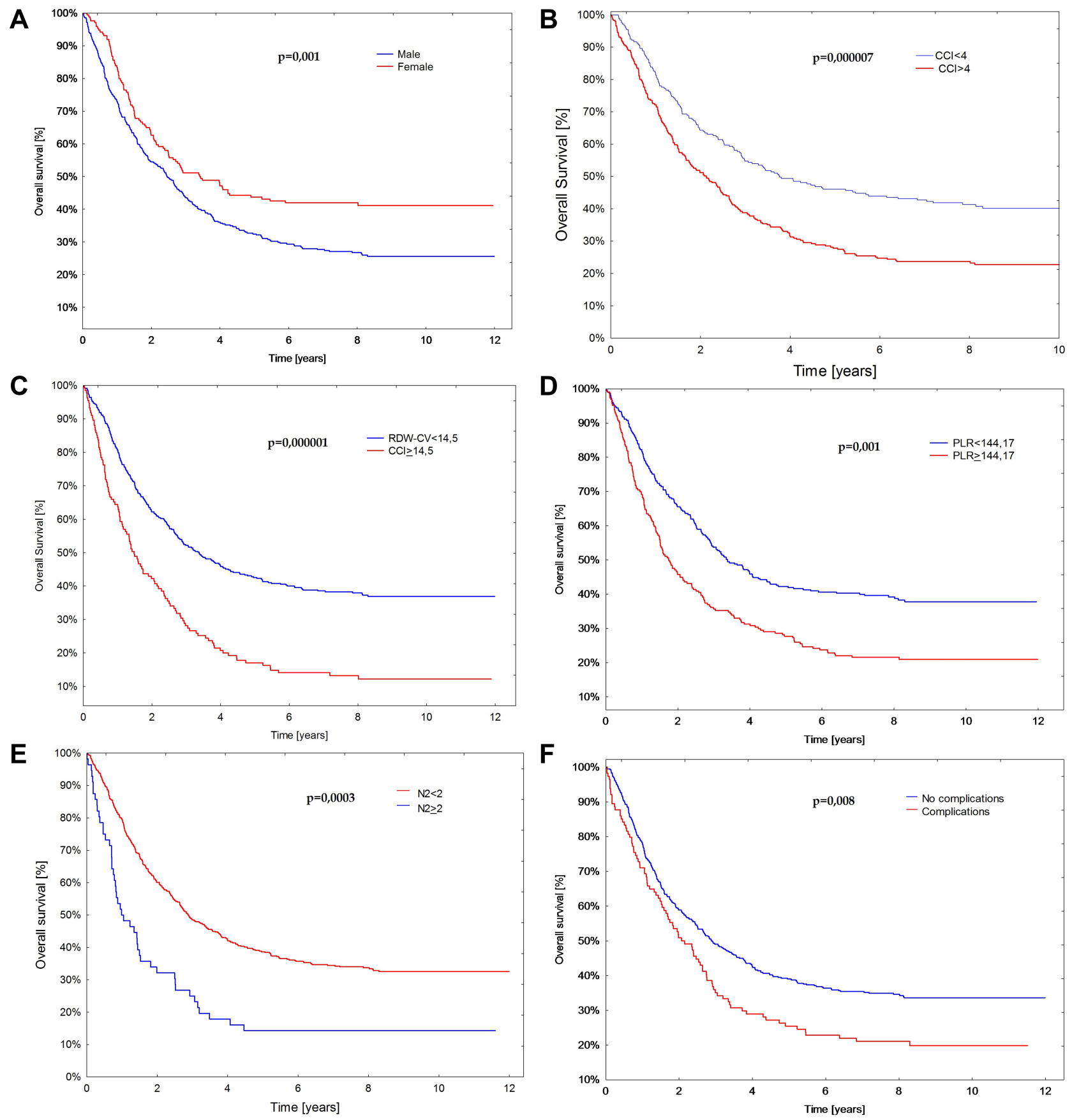

Figure 2 Kaplan-Meier curves of survival of patients operated on due to non-small cell lung cancer. (A) Sex. (B) Charlson Comorbidity Index (CCl). (C) Red cell distribution width-coefficient of variation. (D) Platelet to lymphocyte ratio. (E) The number of involved N2 nodules. (F) Postoperative complications.

is associated with an increased risk of complications and worse prognosis $(\mathrm{p}=0.01){ }^{22}$ In another study, Kos et al did not confirm this prognostic role of RDW-CV. However, the authors themselves stated that the negative result, obtained in their study, might be related to an insufficient number of patients (146) included in the analyses. ${ }^{23}$

Comorbidities undoubtedly affect the natural course of a neoplastic process. $^{24}$ In our study, in order to preoperatively evaluate the patient's condition and determine the mortality rate, we applied the CCI. The multivariate analysis revealed that the $\mathrm{CCI}>4$ is a significant independent negative prognostic factor in NSCLC. Birim et al made a similar observation. They confirmed that CCI values above 3-4 are an independent prognostic factor for the risk of postoperative complications and distant results of treatment. The authors pointed out that CCI is more 
reliable than the individual risk factors assessed in patients and put together. ${ }^{25}$ Maro-Sibilot et al in their study concluded that CCI could be a highly useful parameter for analysing survival of patients operated due to NSCLC. ${ }^{26}$

The patient's general condition and comorbidities are related to an occurrence of postoperative complications ${ }^{24}$ and those have significant implications for the patient's further health condition. ${ }^{27}$ Our analysis revealed that postoperative complications significantly shortened the patients' OS $(p=0.008)$. In the analysed cases as comorbidities, we mostly observed prolonged air leak, atelectasis on the operated site and anaemia which required postoperative blood transfusion. Similar results were obtained by Shinohara et al In their study, they proved that pulmonary complications have a significant effect on OS of patients operated due to NSCLC. ${ }^{28}$ Another study, conducted by Fernandez et al, revealed that the type of complications observed after a pulmonary resection contributes to survival in a particular time period. In the analyzed group of 30,000 patients, the main complications were: atrial fibrillation and pneumonia. $^{29}$ In our analyzed group of patients, atrial fibrillation was not the main complication.

The number of metastatic N2 nodes is the last factor, which in our multivariate analysis significantly determined survival of patients with NSCLC. We observed that more than two metastatic N2 node significantly $(p=0.0002)$ shortens OS. Similar results were obtained by Hanagiri et al, who noted that the number of involved lymphatic nodes is an independent prognostic factor. They emphasised that surgical treatment can be preferred in patients with N2 stage with one or two involved lymphatic nodes. $^{30}$ Also Legras et al confirmed a prognostic value of the number of involved N2 lymphatic nodes, ${ }^{31}$ whereas Sezan et al made conflicting conclusions. ${ }^{32}$

Presented study has a few limitations. The study is a retrospective, patients meeting the operable NSCLC conditions were enrolled. Furthermore the analyzed group included stage IIB and IIIA patients who required adjuvant treatment. This was undoubtedly reflected in the analyzed survival time. Finally, the study group did not have the level of Vit B12, folic acid, and iron measured, which made it impossible to exclude anemia for reasons other than cancer.

\section{Conclusions}

Of parameters available in a panel of laboratory tests and measured in patients operated due to NSCLC, RDW-CW and PLR appear to have a prognostic value. The multivariate analysis confirmed that besides the above parameters, also male sex, CCI $>4$, occurrence of metastases in more than two $\mathrm{N} 2$ group as well as postoperative complications make up independent negative prognostic factors.

The authors of the study believe that to evaluate postoperative prognosis in surgically treated NSCLC patients it is essential to make a comprehensive analysis of various parameters as their mutual correlations might affect a selection of independent prognostic factors.

\section{Disclosure}

All authors declare no conflict of interest.

\section{References}

1. Didkowska J, Wojciechowska U, Mańczuk M, Łobaszewski J. Lung cancer epidemiology: contemporary and future challenges worldwide. Ann Transl Med. 2016;4. doi:10.21037/atm2016.03.11

2. Spiro SG, Silvestri GA. One hundred years of lung cancer. $A m$ J Respir Crit Care Med. 2005;172(5):523-529. doi:10.1164/rccm. 200504-531OE

3. Clark GM. Prognostic factors versus predictive factors: examples from a clinical trial of erlotynib. Mol Oncol. 2008;1(4):406-412. doi:10.1016/j.molonc.2007.12.001

4. Holdenrieder S, Wehnl B, Hettwer K, et al. Carcinoembryonic antigen and cytokeratin-19 fragments for assessment of therapy response in non-small cell lung cancer: a systematic review and meta-analysis. Br J Cancer. 2017;116(8):1037-1045. doi:10.1038/bjc.2017.45

5. Mahar AL, Compton C, McShane LM, et al. Refining prognosis in lung cancer. $J$ Thor Oncol. 2015;10(11):1576-1589. doi:10.1097/ JTO.0000000000000652

6. Itazawa T, Tamaki Y, Komiyama T, et al. The Japan Lung Cancer Society-Japanese Society for Radiation Oncology consensus-based computed tomographic atlas for defining regional lymph node stations in radiotherapy for lung cancer. J Radiat Resp. 2017;58 (1):86-105. doi:10.1093/jrr/rrw076

7. Mountion CF, Dresler CM. Regional lymph node classification for lung cancer staging. Chest. 1997;111(6):1718-1723. doi:10.1378/ chest.111.6.1718

8. Brundage MD, Davies D, Mackillop WJ. Prognostic factors in non-small cell lung cancer a decade of progress. Chest. 2002;122 (3):1037-1057. doi:10.1378/chest.122.3.1037

9. Visnivesky JP, Halm EA. Sex differences in lung cancer survival; do tumors behave differently in elderly women. J Clin Oncol. 2007;25 (13): 1705-1712. doi:10.1200/JCO.2006.081455

10. Ulas A, Tokluoglu S, Kos S, et al. Lung cancer in woman, a different disease; survival differences by sex in Turkey. Asian Pac J Cancer Prev. 2015;16(2):815-822. doi:10.7314/APJCP.2015.16.2.815

11. Song X, Chen D, Yuan M, et al. Total lymphocyte count, neutrophil-lymphocyte ratio, and platelet-lymphocyte ratio as prognostic factors in advanced non-small cell lung cancer with chemoradiotherapy. Cancer Manag Res. 2018;10:6677-6683. doi:10.2147/CMAR.S188578

12. Qiang G, Liang C, Xiao F, et al. Prognostic significance platelet-tolymphocyte ratio in non-small-cell lung cancer: a meta-analysis. Onco Targets Ther. 2016;9:869-876. doi:10.2147/OTT.S96804

13. Sayan M, Kankoc A, Ozkan ND, et al. Simple peripheral blood cell parameters to predict prognosis in non-small cell lung cancer. Indian J Surg. 2020. doi:10.1007/s12262-020-02237-4

14. Lee MB, Rodrigeuz A, Mena G, et al. Platelet-to-lymphocyte ratio and use of NSAIDs during perioperative period as prognostic indicators in patients with NSCLC undergoing surgery. Cancer Control. 2016;23(3):284-294. doi:10.1177/107327481602300312 
15. Xu F, Xu P, Cui W, et al. Neutrophil-to-lymphocyte and platelet-tolymphocyte ratios may aid and identifying patients with non-small cell lung cancer and predicting tumor-node-metastases stage. Oncol Lett. 2018;16:483-489. doi:10.3892/ol.2018.8644

16. Toda $\mathrm{M}$, Tsukioka $\mathrm{T}$, Isumi $\mathrm{N}$, et al. Platelet-to-lymphocyte ratio predict the prognosis of patient with non-small cell lung cancer treated with surgery and postoperative adjuvant chemotherapy. Thorac Cancer. 2018;9(1):112-119. doi:10.1111/1759-7714.12547

17. Yu Y, Qian L, Cui J. Value of neutrophil-to-platelet ratio for predicting lung cancer prognosis: a meta-analysis of 7219 patients. Mol Clin Oncol. 2017;7(3):498-506. doi:10.3892/mco.2017.1342

18. Li J, Yang X, Ma J, et al. Relationship of Red blood cell distribution width with cancer mortality in hospital. Biomed Res Int. 2018:14. doi:10.1155/2018/8914617.

19. Hu L, Li M, Ding Y, et al. Prognostic value of RDW in cancers: a systemic review and meta-analysis. Oncotarget. 2017;8(9):16027-16035. doi:10.18632/oncotarget.13784

20. Koma Y, Onishi A, Matsuoka $\mathrm{H}$, et al. Increased red blood cell distribution width associates with cancer stage and prognosis in patients with lung cancer. PLoS One. 2013;8(11):e8240. doi:10. 1371/journal.pone.0080240

21. Warwick R, Mediratta N, Shackcloth M, et al. Preoperative red cell distribution width in patients undergoing pulmonary resections for non-small-cell lung cancer. Eur J Cardiothorac Surg. 2014;45 (1):108-113. doi:10.1093/ejcts/ezt275

22. Ichinose J, Murakawa T, Kawashima M, et al. Prognostic significance of red cell distribution width in elderly patients undergoing resection for non-small lung cancer. J Thorac Dis. 2016;8(12):3658-3666. doi:10.21037/jtd.2016.12.44

23. Kos M, Hocazade C, Kos FT, et al. Evaluation of the effects of red blood cell distribution width on survival in lung cancer patients. Contemp Oncol (Pozn). 2016;20:153-157. doi:10.5114/wo.2016. 60072
24. Lembicz M, Gabryel P, Brajer-Luftmann B, et al. Comorbidities with non-small cell lung cancer: is there an interdisciplinary consensus needed to qualify patients for surgical treatment. Ann Thorac Med. 2018;13(2):101-107. doi:10.4103/atm.ATM_274_17

25. Birim O, Maat APWM, Kappetein AP, et al. Validation of the Charlson comorbidity index in patients with operated primary non-small cell lung cancer. Eur J Cardiothorac Surg. 2003;23 (1):30-34. doi:10.1016/S1010-7940(02)00721-2

26. Moro-Sibiliot D, Aubert A, Diab S, et al. Comorbidities and Charlson score in resected stage I non-small cell lung cancer. Eur Respir J. 2005;26(3):480-486. doi:10.1183/09031936.05.00146004

27. Cheng X, Chen H. Commentary on the impact of postoperative complications on survival after lung cancer surgery. J Thorac Cardiovasc Surg. 2018;155(3):1265-1266. doi:10.1016/j.jtcvs.2017.10.122

28. Shinohara S, Kobayashi K, Kasahara C, et al. Long-term impact of complications after lung resection in non small cell lung cancer surgery. J Thorac Dis. 2019;11(5):2024-2033. doi:10.21037/jtd.2019.04.91

29. Fernandez FG, Kosinski AS, Furnary AP, et al. Differential effects of operative complications on survival following surgery for primary lung cancer. J Thorac Cardiovasc Surg. 2018;155(3):1254-1264. doi:10.1016/j.jtcvs.2017.09.149

30. Hanagiri T, Takenaka M, Oka S, et al. Clinical significance of the number involved of lymph nodes in patients that underwent surgery for pathological stage III-N2 non- small cell lung cancer. J Cardiothorac Surg. 2011;6(1):144. doi:10.1186/1749-8090-6-144

31. Legras A, Mordant P, Arame A, et al. Long term survival of patients with N2 lung cancer according to the pattern of lymphatic spread. Ann Thorac Surg. 2014;97(4):1156-1162. doi:10.1016/j.athoracsur.2013.12.047

32. Sezen CB, Aksoy Y, Sonmezoglu Y, et al. Prognostic factors for survival in patients with completely resected $\mathrm{pN} 2$ non-small cell lung cancer. Acta Chir Belg. 2019;29:1-7. doi:10.1088/00015458. 2019.1658355

\section{Publish your work in this journal}

Cancer Management and Research is an international, peer-reviewed open access journal focusing on cancer research and the optimal use of preventative and integrated treatment interventions to achieve improved outcomes, enhanced survival and quality of life for the cancer patient.
The manuscript management system is completely online and includes a very quick and fair peer-review system, which is all easy to use. Visit http://www.dovepress.com/testimonials.php to read real quotes from published authors. 